Accretion Phenomena and Related Outflows, IAU Colloquium 169

ASP Conference Series, Vol. 121, 1997

D.T. Wickramasinghe, L. Ferrario, and G.V. Bicknell, eds.

\title{
Periodical Instabilities in the Blackhole Candidate GX339-4 Accretion Disk
}

\author{
Philippe Durouchoux \\ Service d'Astrophysique, C.E. Saclay, 91191 Gif sur Yvette Cedex, \\ FRANCE
}

\begin{abstract}
GX339-4, a black hole candidate, has been monitored since more than 30 years by X and gamma-ray spectrometers on board a dozen of satellites. One of the main characteristics of this source is its bimodal behaviour (high and low states). These states, apparently driven by accretion of matter from a companion onto a compact object,appear to be periodic, with a 14.5 month repetitivity, and an approximative 4 month duration. We discuss, in this paper, on periodic instabilities in the accretion disk surrounding GX339-4 to explain this bimodal behaviour.
\end{abstract}

